\title{
ERP B3: Business Continuity Service Level Agreement Translation and Optimisation ${ }^{\star}$
}

\author{
Ulrich Winkler and Wasif Gilani \\ SAP Research, \\ The Concourse, Queen's Road, Belfast BT3 9DT, United Kingdom \\ \{ulrich.winkler, wasif.gilani\}@sap.com \\ http://www.sap.com/research
}

\begin{abstract}
ERP B3 is a SLA@SOI framework based solution for Service Level Agreements driven, on demand and dynamic provisioned business applications. We extended the ERP B3 framework with a process-centric Business Continuity Analysis toolkit. In this demo we want to show how our toolkit helps Business Continuity Manager (a) to estimate business impact of failed IT services (b) to validate SLAs and verify if selected SLAs are appropriated and (c) to determine an optimal set of servicelevel SLAs to minimise business disruptions.
\end{abstract}

Keywords: SLA, SLA@SOI, SLA SLA translation, SLA planning, Business Continuity Management, BCM.

\section{Introduction}

The SLA@SOI framework provides comprehensive support for holistic and transparent SLA management, SLA translation and SLA negotiation [12. Lessons learned from using the SLA@SOI framework to implement ERP B3 solution to perform IT centric SLA translation are discussed in in [3].

We extended ERP B3 and added components which helps Business Continuity Manager (a) to estimate business impact of failed IT services (b) to translate business level requirements to IT-service and facility-level requirements and (c) to select an optimal set of service-level and facility-level SLAs [4. The overall objective is to minimise business disruptions.

Our toolkit is very interactive and supports the Business Continuity Manager to explore potential alternatives and to answer "what-if-questions". The best way to explain these interactive modelling and optimisation components of our toolkit is with a demonstration.

\footnotetext{
* The research leading to these results is partially supported by the European Community's Seventh Framework Programme (FP7/2001-2013) under grant agreement no. 216556 .
} 


\section{Outline of the ERP B3 BCM Demonstration}

1. BCM: We will educate the audience about business process modelling, business performance analysis, and the role and importance of Business Continuity Management.

2. Business Impact: We extended a process modelling tool with business impact annotation modelling support. We will demonstrate how a business continuity manager uses this impact modelling support to model financial, legal and other business requirements in a process-centric way.

3. Dependency and Risk Modelling: We designed and implemented a dependency and risk modelling tool to model the IT service and facility hierachy. This tool enables the continuity manager to annotate risk to the service hierachy.

4. SLA translation, selection and validation: We explain how the continuity manager links the service hierarchy to business activities. Once this linkage has been established our tool translates business level requirements down to service level requirements and verifies if selected SLAs are suitable to fulfil business level requirements.

5. SLA optimisation: If more than one SLA has to be selected the SLA selection and validation problem becomes an optimisation problem. We explain how our tool solves this problem and proposes an optimal set of SLAs.

\section{Conclusions}

ERP B3 makes use of the SLA@SOI framework to provide service level aware ondemand business applications. In this demo we show how we extended the ERP B3 framework with Business Continuity Analysis support. We show how a continuity manager uses our extension to translate business level requirements down to service level requirements. An optimisation components helps the continuity manager to select a set of optimal SLAs.

\section{References}

1. SLA@SOI project: IST-216556; Empowering the Service Economy with SLA-aware Infrastructures, http://www.sla-at-soi.eu/

2. Theilmann, W., Happe, J., Kotsokalis, C., Edmonds, A., Kearney. K.: A Reference Architecture for Multi-Level SLA Management. Journal of Internet Engineering (2010) (to appear)

3. Theilmann, W., Winkler, U., Happe, J., de Abril, I.M.: Managing On-Demand Business Applications with Hierarchical Service Level Agreements. In: Berre, A.J., Gómez-Pérez, A., Tutschku, K., Fensel, D. (eds.) FIS 2010. LNCS, vol. 6369, pp. 97-106. Springer, Heidelberg (2010)

4. Winkler, U., Gilani, W., Fritzsche, M., Marshall, A.: Model-Driven Framework for Process-centric Business Continuity Management. In: Seventh International Conference on the Quality of Information and Communications Technology, Porto, pp. 248-252 (2010) 